\title{
Convolution Surfaces for Line Skeletons with Polynomial Weight Distributions
}

\author{
Xiaogang Jin \\ State Key Lab of CAD\&CG, Zhejiang University \\ Chiew-Lan Tai \\ Hong Kong University of Science and Technology \\ Jieqing Feng, Qunsheng Peng \\ State Key Lab of CAD\&CG, Zhejiang University
}

\begin{abstract}
Convolution surfaces generalize point-based implicit surfaces to incorporate higherdimensional skeletal elements, of which line segments can be considered the most fundamental since they can approximate curve skeletons. Existing analytical models for line-segment skeletons assumes uniform weight distributions and thus can only produce constant-radius convolution surfaces. This paper presents an analytical solution for convolving line-segment skeletons with a variable kernel modulated by a polynomial function, thus allowing generalized cylindrical convolution surfaces to be conveniently modeled. Its computation requirement is competitive compared with the case of uniform weight distribution. The source code of field computation is available online.
\end{abstract}

\section{Introduction}

Implicit surface has been proven to be a good representation for modeling and animating smooth deformable objects of complex topology that may change over time, such as liquid, snow, cloud and organic shapes [Bloomenthal et al. 97], [Dobashi et al. 00], [Cani, Desbrun 97], [Nishita et al. 97], [Jin et al. 00]. A skeleton-based implicit surface is most commonly defined as an iso-surface satisfying the following equation:

$$
S=\left\{(x, y, z) \mid \sum F_{i}(x, y, z)-T=0\right\},
$$

where $F_{i}$ is the field function of the $i$ th contribution source and $T$ is the threshold field value. For example, the implicit functions in metaballs (or blobs, soft objects) [Blinn 82], [Nishimura et al. 85], [Wyvill et al. 86], [Wyvill, Wyvill 89] are defined as a summation of point fields. Metaballs are widely implemented in commercial software packages (e.g., Softimage, 3D Studio Max), but they suffer from a major drawback in being inadequate for representing flat surfaces and generalized cylinders. Distance surface allows the use of higher-dimensional skeletons [Bloomenthal, Wyvill 90], [Bloomenthal 95], however creases or curvature discontinuity may arise when there are multiple non-convex skeletal primitives.

Bloomenthal and Shoemake [Bloomenthal, Shoemake 91] presented convolution surfaces as a natural and powerful extension to point-based field surfaces. By convolving skeletons with a three-dimensional low-pass Gaussian filter kernel, the 
resulting iso-surfaces overcome the above-mentioned drawback of distance surfaces [Bloomenthal 97]. The skeletal elements in convolution surfaces can be points, line segments, curves, polygons and other geometric primitives. As skeletons are natural abstractions for shapes, convolution surfaces offer a convenient means of controlling the shape of the underlying modeling object.

While the modeling potentials of convolution surfaces are very attractive, their mathematical formulation still poses some open problems, stemming from the fact that the convolution integrals seldom yield closed-form solutions that can be directly evaluated. The possibility of deriving closed form solutions depends on both the kernel function and the skeletal element. Bloomenthal and Shoemake [Bloomenthal, Shoemake 91] calculated the field function numerically using a point-sampling method, which, unfortunately, suffers from potential under-sampling artifacts. By employing a new kernel function-Cauchy function-McCormack and Sherstyuk [McCormack, Sherstyuk 98], [Sherstyuk 99] deduced analytical solutions for the following elements: point, line segment, polygon, arc and plane. Their method thus provides an accurate and robust solution for more general-shape convolution surfaces.

The line-segment analytical model derived by McCormack and Sherstyuk assumes uniform weight distribution, and produces only constant-radius convolution surfaces around the line-segment skeleton. Modeling surfaces of varying radius thus requires specifying multiple line segments. To overcome this problem, this paper presents a closed-form model for line-segment skeletons with weight distribution modulated by a polynomial. Since many objects can be abstracted as curve skeletons, which can in turn be approximated by line segments, our closed-form solution can facilitate the modeling of a large variety of generalized cylindrical shapes ${ }^{1}$.

\section{Convolution Surface}

A convolution surface is an iso-surface in a scalar field implicitly defined by a skeleton consisting of three-dimensional points, and a potential function representing the contribution of each skeletal point to the scalar field. In this paper, we adopt the following convolution surface definition given by McCormack and Sherstyuk [McCormack, Sherstyuk 98]. Let $\mathbf{P}(x, y, z)$ be a space point in $R^{3}$, and let $g: R^{3} \rightarrow R$ be the geometry function that represents a modeling skeleton $V$ :

$$
g(\mathbf{P})=\left\{\begin{array}{cc}
1, & \mathbf{P} \in \text { skeleton } V \\
0, & \text { otherwise }
\end{array}\right.
$$

Let $f: R^{3} \rightarrow R$ be a potential function generated by a single point in the skeleton $V$, and let $\mathbf{Q}$ be a point in the skeleton, then the total field contributed by the skeleton at a point $\mathbf{P}$ is the convolution of two functions $f$ and $g$,

\footnotetext{
${ }^{1}$ Grimm [Grimm 99] has also investigated implicit generalized cylinders; her method involves specifying an axis and one or more profile curves. In contrast, our method produces exact convolution surfaces and extends the versatility of modeling with convolution surfaces.
} 


$$
F(\mathbf{P})=\int_{V} g(\mathbf{Q}) f(\mathbf{P}-\mathbf{Q}) d V=(f \otimes g)(\mathbf{P}),
$$

Thus, $f$ is also called the convolution kernel. For convenience, we rewrite the field function of the convolution surface as the volume integral along the skeleton,

$$
F(\mathbf{P})=\int_{V} f(\mathbf{P}-\mathbf{Q}) d V .
$$

In this paper, we adopt the Cauchy kernel function proposed by McCormack and Sherstyuk [McCormack, Sherstyuk 98],

$$
f(\mathbf{P}-\mathbf{Q})=\frac{1}{\left(1+s^{2} r^{2}\right)^{2}},
$$

where $r=\|\mathbf{P}-\mathbf{Q}\|$, and $s$ is a parameter for controlling the width of the kernel. The field function $F(\mathbf{P})$ now becomes

$$
F(\mathbf{P})=\int_{V} \frac{d V}{\left(1+s^{2} r^{2}\right)^{2}}
$$

Superposition is one of the most important properties of convolution surfaces; that is, summing the convolution surfaces generated by two separate skeletons yields the same surface as that generated by their combined skeleton. It is this property that enables convolution surfaces to overcome the problem of bulges and creases in distance surfaces. This independent evaluation feature guarantees that the user only has to be concerned with the shape of the skeleton, not the number of segments used, when designing a convolution surface. Superposition property ensures that the skeletons can be arbitrarily subdivided and the field function of the sub-skeletons can be simply summed to evaluate the final convolution surface.

\section{Line-Segment Skeleton with Polynomial Weight Distributions}

We multiply the field function at a point $\mathbf{Q}$ by $q(\mathbf{Q}): R^{3} \rightarrow R$ to define a weighted convolution surface model with non-uniform weight distributions:

$$
F(\mathbf{P})=\int_{V} q(\mathbf{Q}) f(\mathbf{P}-\mathbf{Q}) d V=\int_{V} \frac{q(\mathbf{Q}) d V}{\left(1+s^{2} r^{2}\right)^{2}} .
$$

We now derive the analytical formulae for line-segment skeleton with polynomial weight distributions. A line segment of length $l$, with start point $\mathbf{b}$ and unit direction $\mathbf{a}$, can be represented parametrically as

$$
\mathbf{L}(t)=\mathbf{b}+t \mathbf{a}, \quad 0 \leq t \leq l
$$

Letting $\mathbf{d}=\mathbf{P}-\mathbf{b}$, the squared distance from a point $\mathbf{P}$ to a point on the line $\mathbf{L}(t)$ is then 


$$
r^{2}(t)=d^{2}+t^{2}-2 t \mathbf{d} \bullet \mathbf{a}
$$

where $d=\|\mathbf{d}\|$. Let $F_{\text {line }}^{t^{i}}(\mathbf{P})$ denote the field function of the line segment $\mathbf{L}(t)$ with weight distribution $t^{i}$; for $i=0,1,2,3$, we obtain

$$
\begin{array}{ll}
F_{\text {line }}^{1}(\mathbf{P})=\int_{0}^{l} \frac{d t}{\left(1+s^{2} r^{2}(t)\right)^{2}}, & F_{\text {line }}^{t}(\mathbf{P})=\int_{0}^{l} \frac{t d t}{\left(1+s^{2} r^{2}(t)\right)^{2}}, \\
F_{\text {line }}^{t^{2}}(\mathbf{P})=\int_{0}^{l} \frac{t^{2} d t}{\left(1+s^{2} r^{2}(t)\right)^{2}}, & F_{\text {line }}^{t^{3}}(\mathbf{P})=\int_{0}^{l} \frac{t^{3} d t}{\left(1+s^{2} r^{2}(t)\right)^{2}} .
\end{array}
$$

By substituting $r^{2}(t)$ into $F_{\text {line }}^{1}(\mathbf{P})$, letting $h=\mathbf{d} \bullet \mathbf{a}$, and integrating, we obtain

$$
\begin{aligned}
F_{\text {line }}^{1}(\mathbf{P}) & =\int_{0}^{l} \frac{d t}{\left(1+\left(s^{2}(t-h)^{2}+s^{2} d^{2}-s^{2} h^{2}\right)\right)^{2}} \\
& =\frac{1}{s^{4}} \int_{-h}^{l-h} \frac{d t}{\left(t^{2}+(p / s)^{2}\right)^{2}} \\
& =\frac{1}{2 p^{2}}\left[\frac{h}{s^{2} h^{2}+p^{2}}+\frac{l-h}{s^{2}(l-h)^{2}+p^{2}}\right]+\frac{1}{2 s p^{3}}\left(\operatorname{atan}\left[\frac{s h}{p}\right]+\operatorname{atan}\left[\frac{s(l-h)}{p}\right]\right),
\end{aligned}
$$

where $p$ is a distance term:

$$
p^{2}=1+s^{2}\left(d^{2}-h^{2}\right)
$$

Similarly, we can derive the analytical formulae for $F_{\text {line }}^{t}(\mathbf{P}), F_{\text {line }}^{t^{2}}(\mathbf{P}), F_{\text {line }}^{t^{3}}(\mathbf{P})$ as follows:

$$
\begin{aligned}
F_{\text {line }}^{t}(\mathbf{P}) & =\int_{0}^{l} \frac{t d t}{\left(s^{2}(t-h)^{2}+p^{2}\right)^{2}} \\
& =\frac{1}{2 s^{2}}\left[\frac{1}{s^{2} h^{2}+p^{2}}-\frac{1}{s^{2}(l-h)^{2}+p^{2}}\right]+h F_{\text {line }}^{1}(\mathbf{P}) \\
F_{\text {line }}^{t^{2}}(\mathbf{P}) & =\int_{0}^{l} \frac{t^{2} d t}{\left(s^{2}(t-h)^{2}+p^{2}\right)^{2}} \\
& =-\frac{1}{2 s^{2}}\left[\frac{h}{s^{2} h^{2}+p^{2}}+\frac{l-h}{s^{2}(l-h)^{2}+p^{2}}\right]+\frac{1}{2 p s^{3}}\left(\operatorname{atan}\left[\frac{s h}{p}\right]+\operatorname{atan}\left[\frac{s(l-h)}{p}\right]\right. \\
& -h^{2} F_{\text {line }}^{1}(\mathbf{P})+2 h F_{\text {line }}^{t}(\mathbf{P}) \\
& =\frac{1}{s^{3} p}\left(\operatorname{atan}\left[\frac{s h}{p}\right]+\operatorname{atan}\left[\frac{s(l-h)}{p}\right]\right)-\left(h^{2}+(p / s)^{2}\right) F_{\text {line }}^{1}(\mathbf{P})+2 h F_{\text {line }}^{t}(\mathbf{P}),
\end{aligned}
$$




$$
\begin{aligned}
F_{\text {line }}^{t^{3}}(\mathbf{P})= & \int_{0}^{l} \frac{(t-h)^{3} d t}{\left(s^{2}(t-h)^{2}+p^{2}\right)^{2}}+h^{3} F_{\text {line }}^{1}(\mathbf{P})-3 h^{2} F_{\text {line }}^{t}(\mathbf{P})+3 h F_{\text {line }}^{t^{2}}(\mathbf{P}) \\
= & \frac{1}{2 s^{4}}\left[\ln \frac{s^{2}(l-h)^{2}+p^{2}}{s^{2} h^{2}+p^{2}}\right]-\frac{p^{2}}{2 s^{4}}\left[\frac{1}{s^{2} h^{2}+p^{2}}-\frac{1}{s^{2}(l-h)^{2}+p^{2}}\right] \\
& +h^{3} F_{\text {line }}^{1}(\mathbf{P})-3 h^{2} F_{\text {line }}^{t}(\mathbf{P})+3 h F_{\text {line }}^{t^{2}}(\mathbf{P}) .
\end{aligned}
$$

Analogously, analytical formulae for $F_{\text {line }}^{t^{i}}(\mathbf{P})$ with $i \geq 4$ can be derived; however, since cubic polynomials are usually sufficient for meeting user requirements, we omit these formulae here. Note that an $F_{\text {line }}^{t^{i}}(\mathbf{P}), i \geq 1$ can be represented in terms of $F_{\text {line }}^{t^{i-1}}(\mathbf{P}), F_{\text {line }}^{t^{i-2}}(\mathbf{P}), \cdots, F_{\text {line }}^{1}(\mathbf{P}) ;$ this enables the use of incremental calculations which are more efficient.

Based on the closed-form field functions of a line segment with weight distributions $1, t, t^{2}, t^{3}$, we can now use a cubic polynomial function to define a profile distribution function along the skeletal line segment. To provide an intuitive interface for controlling the cubic curve, we represent it in the Bezier form with control points $\left(j / 3, q_{j}\right)$ where $j=0, \cdots, 3$. This form of Bezier curve can simply be rewritten as $q(u)=\sum q_{j} B_{j}(u)$ where $B_{j}(u)$ are cubic Bernstein basis functions [Farin97]. The designed profile Bezier curve is then converted to power basis form.

For linear weight distribution, which is the most frequently used in practice, the formula is very simple. Let the weights at the start and end points of the line segment $\mathbf{L}(t)$ be $\tilde{q}_{0}$ and $\tilde{q}_{1}$ respectively; then the weight at parameter $t$ is

$$
q(t)=\tilde{q}_{0}+\frac{\tilde{q}_{1}-\tilde{q}_{0}}{l} t
$$

and the field function of the entire line segment is

$$
F_{\text {line }}(\mathbf{P})=\tilde{q}_{0} F_{\text {line }}^{1}(\mathbf{P})+\frac{\tilde{q}_{1}-\tilde{q}_{0}}{l} F_{\text {line }}^{t}(\mathbf{P}) .
$$

We now derive the field function of a skeletal polyline $\mathbf{P}_{0} \mathbf{P}_{1} \cdots \mathbf{P}_{n}$ with linear weight distributions, given that the weights at $\mathbf{P}_{0}$ and $\mathbf{P}_{n}$ are $\tilde{q}_{0}$ and $\tilde{q}_{1}$ respectively. Let the length of the $i$ th segment $\mathbf{P}_{i} \mathbf{P}_{i+1}$ be $l_{i}=\left\|\mathbf{P}_{i+1}-\mathbf{P}_{i}\right\|, i=0,1, \cdots, n-1$, and denote the field functions along the line segment $\mathbf{P}_{i} \mathbf{P}_{i+1}$ with weight distributions $1, t$, as 
${ }^{i} F_{\text {line }}^{1}(\mathbf{P}),{ }^{i} F_{\text {line }}^{t}(\mathbf{P})$ respectively. It is easy to derive the weight at point $\mathbf{P}_{i}$, denoted by $q_{i}$, as follows:

$$
q_{i}=\tilde{q}_{0}+\left(\sum_{j=0}^{i-1} l_{j} / \sum_{j=0}^{n-1} l_{j}\right)\left(\tilde{q}_{1}-\tilde{q}_{0}\right) .
$$

Thus, the field function for the entire polyline is

$$
F_{\text {polyline }}(\mathbf{P})=\sum_{i=0}^{n-1}\left\{q_{i}{ }^{i} F_{\text {line }}^{1}(\mathbf{P})+\frac{\left(q_{i+1}-q_{i}\right)}{l_{i}}{ }^{i} F_{\text {line }}^{t}(\mathbf{P})\right\} \text {. }
$$
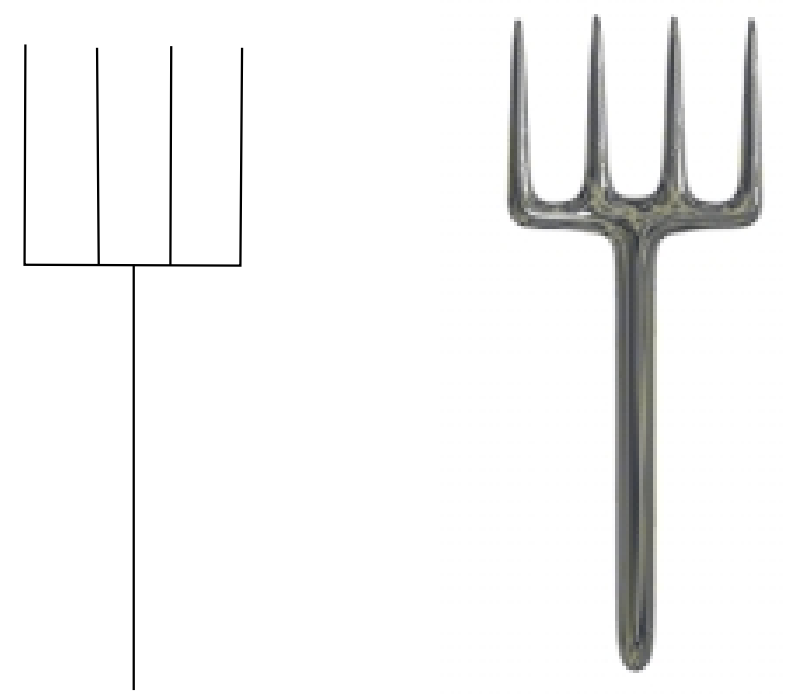

Figure 1. Hayfork. (a) Skeleton. (b) Convolution surface.
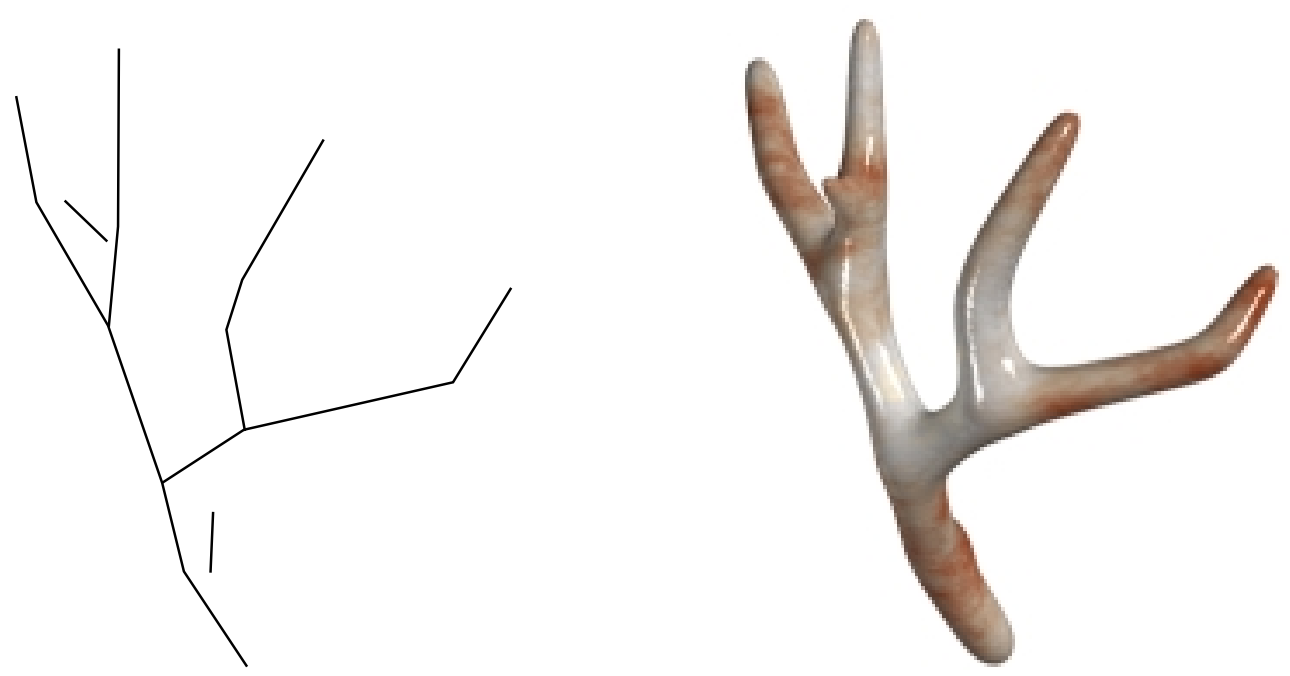

Figure 2. Deer horn. (a) Skeleton. (b) Convolution surface. 

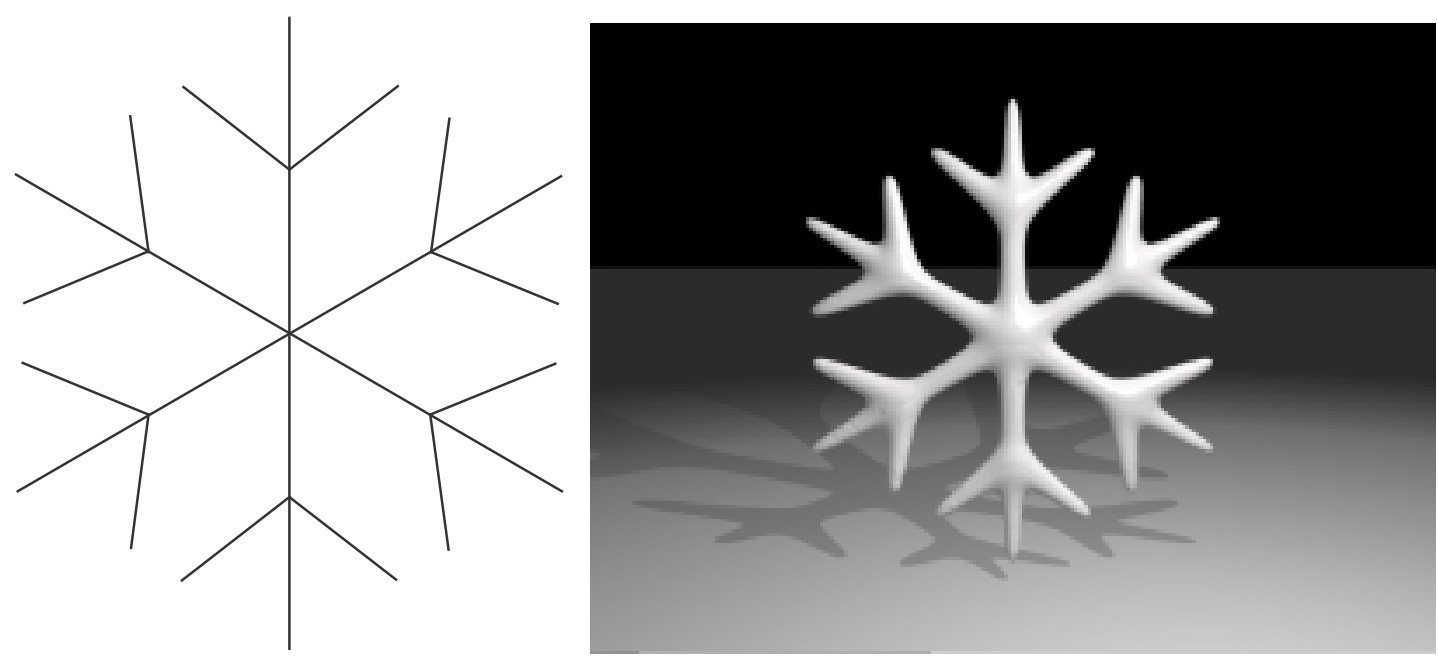

Figure 3. Snowflake. (a) Skeleton. (b) Convolution surface.
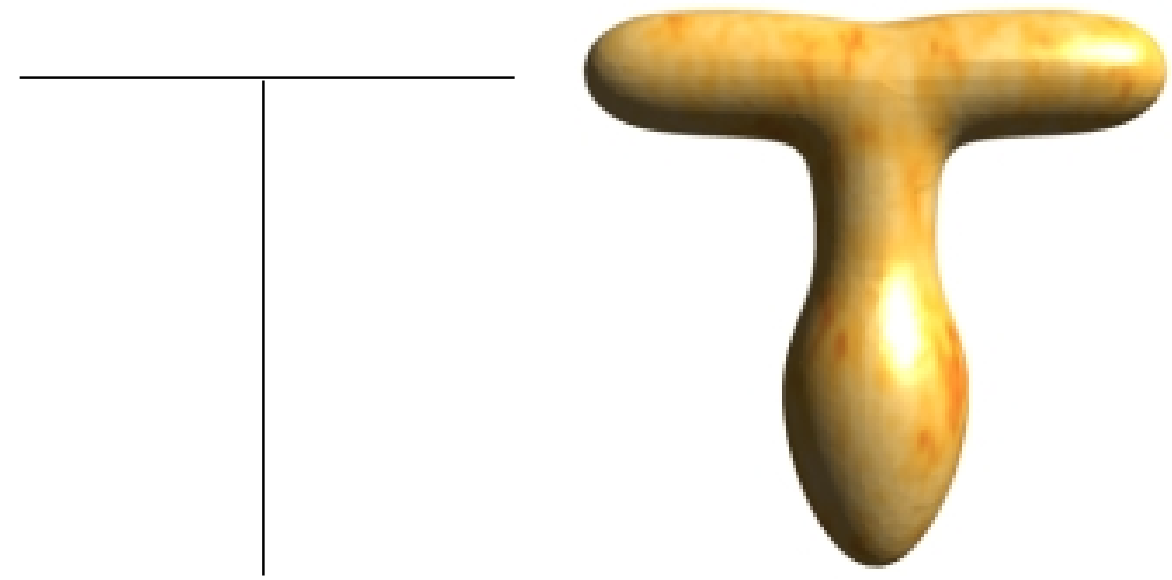

Figure 4. T-shape object. (a) Skeleton. (b) Convolution surface.

\section{Examples and Performance}

To demonstrate the modeling capabilities of our method, we have modeled some examples. For uniform processing, all the convolution models are first polygonized into polygon meshes, which are then ray-traced with solid or projective texture mapping. Figures 1 to 3 are convolution surfaces that adopt linear weight distributions. The underlying skeletal representation is shown on the left of each example. The hayfork, deer horn and snowflake are respectively modeled using 6,15 and 18 line segments. Figure 4 shows an example that uses a cubic polynomial distribution for the vertical line segment.

For more complex examples, we show a maple tree in Figure 5 whose stem and main branches are modeled using polyline skeletons with linear weight functions (the twigs and leaves are modeled using traditional modeling methods). Similarly, the branches of the potted plant in Figure 6 are modeled in a similar way (the leaves are modeled separately before adding to the branches). These examples demonstrate the convenience of modeling convolution surfaces of generalized cylindrical shapes using our analytical models. 


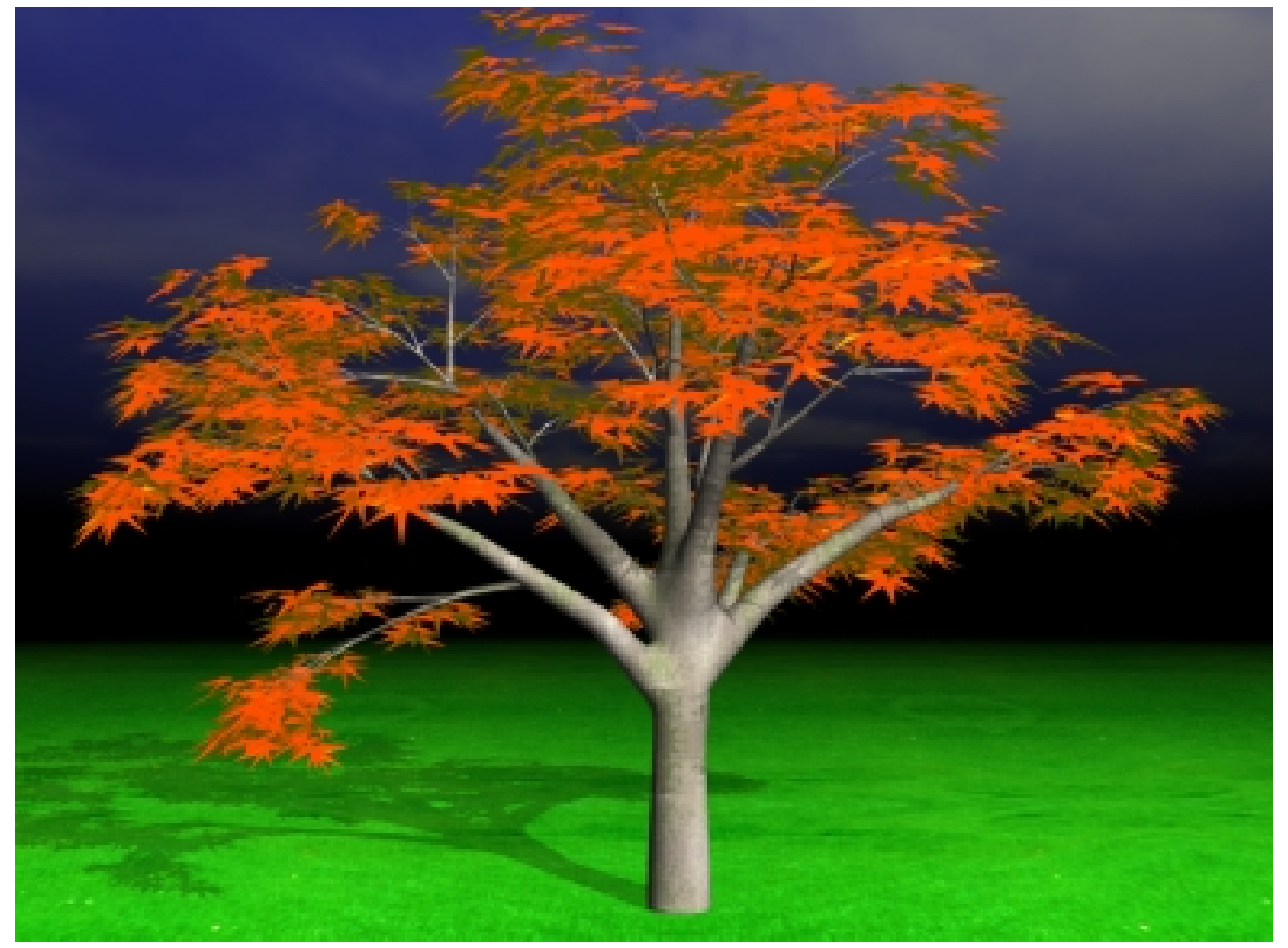

Figure 5. Maple tree.

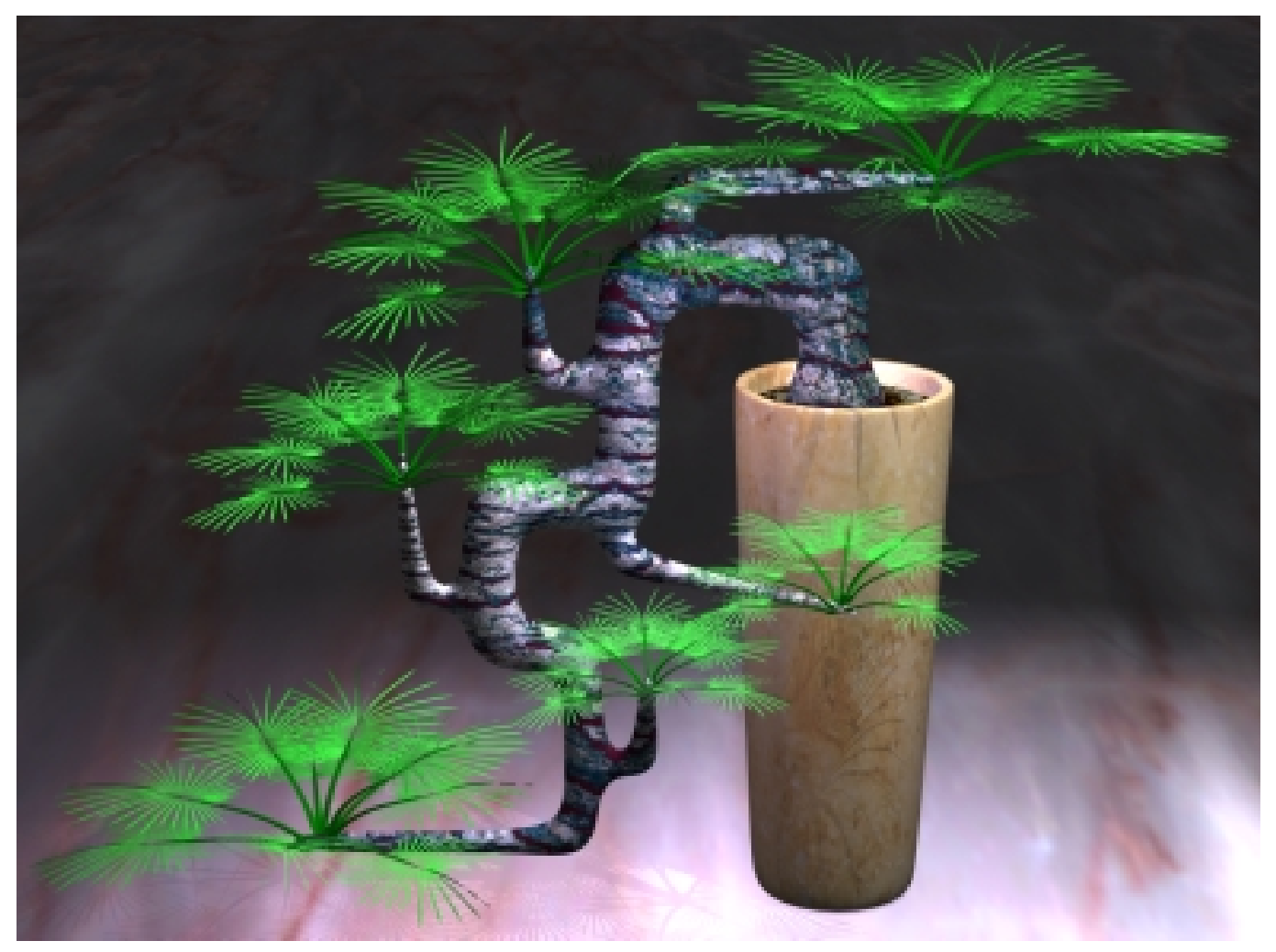

Figure 6. Potted plant.

Tables 1 and 2 show the computational efficiency of our algorithm. Table 1 gives the number of special function calls and floating-point operations required in calculating 
the field function for a line segment with constant distribution; optimizations have been performed to reduce the number of operations. Table 2 gives the incremental operations required for a line segment with weight distributions $t, t^{2}, t^{3}$. From these tables, we can conclude that the incremental cost from constant distribution to linear distribution is nominal—only additional five multiplications/divisions and two additions/subtractions are needed. Even for cubic polynomial distribution, the additional computation cost is only one $\ln$ operation, 24 multiplications/divisions, and 10 additions/subtractions, which is less than twice the cost of constant distribution.

\begin{tabular}{|c|c|c|c|c|c|c|c|}
\hline \multirow{2}{*}{ Weight distribution } & \multicolumn{7}{|c|}{ Special functions and floating-point operations } \\
\cline { 2 - 9 } & atan & sqrt & $\ln$ & $*$ & $/$ & + & - \\
\hline 1 & 2 & 1 & 0 & 17 & 5 & 10 & 5 \\
\hline
\end{tabular}

Table 1. Computational costs for a line segment convolved with constant distribution.

\begin{tabular}{|c|c|c|c|c|c|c|c|}
\hline \multirow{2}{*}{ Weight distribution } & \multicolumn{6}{|c|}{ Incremental special functions and floating-point operations } \\
\cline { 2 - 8 } & atan & sqrt & $\ln$ & $*$ & $/$ & + & - \\
\hline$t$ & 0 & 0 & 0 & 2 & 3 & 1 & 1 \\
\hline$t^{2}$ & 0 & 0 & 0 & 4 & 2 & 2 & 1 \\
\hline$t^{3}$ & 0 & 0 & 1 & 8 & 5 & 2 & 3 \\
\hline
\end{tabular}

Table 2. Incremental computational costs for a line segment convolved with $t, t^{2}, t^{3}$ distribution.

\section{Discussion}

Skeleton-based convolution surface modeling can create and animate a wide variety of complex objects, which maybe difficult with parametric geometric modeling methods. Since curve skeletons are good abstractions for a wide variety of natural forms, and curve skeletons can be approximated by polylines, our method is considerably general in its applicability. Combining with other skeletal elements, our method facilitates the modeling of trees, sea-forms and organic. By defining tapering factors in grammar rules, our method can also be easily incorporated into string rewriting systems (L-systems) to describe the geometric shapes of plant trunks [Prusinkiewicz, Lindenmayer 90].

Since convolution surfaces are iso-surfaces that are determined by skeletons, profile curves, and threshold field value, our method has the following limitations. (1) Unlike traditional sweeping operations, the profile curve only approximates that of the generated generalized cylinder because the profile curve in general does not lie on the iso-surface; thus precise specification of the radius of the generalized cylinder is difficult. (2) If the threshold exceeds the field of some part of a skeleton, no convolution surface is produced at that part; this requires special attention and causes inconveniences in some applications. 


\section{Acknowledgements}

Part of this research work was conducted while the first author was a visiting researcher at the Hong

Kong University of Science and Technology. This work received support from Hong Kong Research Grant Council (HKUST6215/99E), National Natural Science Foundation of China (grant No. 69973040), Zhejiang Provincial Natural Science Foundation (Grant No. 698022) and Innovative Research Groups (Grant No. 60021201). The authors are grateful to Andrei Sherstyuk for his help and discussions on convolution surfaces.

\section{References}

[Blinn 82] J. Blinn. "A Generalization of Algebraic Surface Drawing." ACM Transactions on Graphics, 1(3): 235-256 (1982).

[Bloomenthal 95] J. Bloomenthal. Skeletal Design of Natural Forms. Ph.D thesis, University of Calgary, Dept. of Computer Science, 1995.

[Bloomenthal 97] J. Bloomenthal. "Bulge Elimination in Convolution Surfaces." Computer Graphics Forum, 16(1): 31-41 (1997).

[Bloomenthal et al. 97] J. Bloomenthal, C. Bajaj, J. Blinn, M. Cani, A. Rockwood, B. Wyvill and G. Wyvill. An Introduction to Implicit Surfaces. Los Altos, CA: Morgan Kaufmann Publishers, 1997.

[Bloomenthal, Shoemake 91] J. Bloomenthal, K. Shoemake. "Convolution Surface." Computer Graphics (Proc. SIGGRAPH'91), 25(4): 251-256 (1991).

[Bloomenthal, Wyvill 90] J. Bloomenthal and B. Wyvill. "Interactive Techniques for Implicit Modeling." Computer Graphics, 24(2): 109-116 (1990).

[Cani, Desbrun 97] M. Cani and M. Desbrun. "Animation of Deformable Models Using Implicit Surfaces." IEEE Transactions on Visualization and Computer Graphics, 3(1):39-50 (1997).

[Dobashi et al. 00] Y. Dobashi, K Kaneda, H.Yamashita, T. Okita and T. Nishita. "A Simple, Efficient Method for Realistic Animation of Clouds." In Proc. of SIGGRAPH'00, Computer Graphics Proceedings, Annual Conference Series, edited by Kurt Akeley, pp.19-28, Reading, MA: Addison Wesley, 2000.

[Farin 97] G. Farin. Curves and Surfaces for Computer Aided Geometric Design: A Practical Guide. New York: Academic Press, Fourth Edition, 1997.

[Grimm 99] C. Grimm. "Implicit Generalized Cylinders using Profile Curves." In Proc. of Implicit Surfaces'99, pp.33-41, Bordeaux, France, 1999.

[Jin et al. 00] X. Jin, Y. Li and Q. Peng. "General Constrained Deformations Based on Generalized Metaballs." Computers \& Graphics, 24(2):219-231 (2000).

[McCormack, Sherstyuk 98] J. McCormack and A. Sherstyuk. "Creating and Rendering Convolution Surfaces.” Computer Graphics Forum, 17(2): 113-120 (1998).

[Nishimura et al. 85] H. Nishimura, M. Hirai and T. Kawai. "Object Modeling by Distribution Function and a Method of Image Generation." Transactions on IECE, 68-D(4):718-725 (1985)

[Nishita et al. 97] T. Nishita, H. Iwasaki,Y. Dobashi and E. Nakamae. "A Modeling and Rendering Method for Snow by Using Metaballs.” Computer Graphics Forum, 16(3):357-364 (1997).

[Prusinkiewicz, Lindenmayer 90] P. Prusinkiewicz and A. Lindenmayer. The algorithmic Beauty of Plants. New York: Springer-Verlag, 1990. 
[Sherstyuk 99] A. Sherstyuk. "Kernel Functions in Convolution Surfaces: A Comparative Analysis." The Visual Computer, 15(4):171-182 1999.

[Wyvill et al. 86] G. Wyvill, C. McPheeters and B. Wyvill. "Data Structure for Soft Objects." The Visual Computer, 2(4):227-234 (1986).

[Wyvill, Wyvill 89] B. Wyvill and G. Wyvill. "Field Functions for Implicit Surfaces." The Visual Computer, 5(1/2):75-82 (1989).

\section{Web Information:}

The $\mathrm{C}$ source code of the field computation and images are available online at http: / /www.acm.org/jgt/papers/JinEtAl01

Xiaogang Jin, State Key Laboratory of CAD\&CG, Zhejiang University, Hangzhou, 310027, P.R. China. (jin@cad.zju.edu.cn)

Chiew-Lan Tai, Department of Computer Science, Hong Kong University of Science and Technology, Clear Water Bay, Kowloon, Hong Kong. http://www.cs.ust.hk/ taicl/ (taicl@cs.ust.hk)

Jieqing Feng, State Key Laboratory of CAD\&CG, Zhejiang University, Hangzhou, 310027, P.R. China. (jqfeng@cad.zju.edu.cn)

Qunsheng Peng, State Key Laboratory of CAD\&CG, Zhejiang University, Hangzhou, 310027, P.R. China. (peng@cad.zju.edu.cn) 